\title{
Ground-water monitoring using polymer optical fibre Bragg gratings
}

\section{Mehravar, H. Yang, Wei Zhang, D. J. Webb}

M. Mehravar, H. Yang, Wei Zhang, D. J. Webb, "Ground-water monitoring using polymer optical fibre Bragg gratings," Proc. SPIE 11199, Seventh European Workshop on Optical Fibre Sensors, 111991M (28 August 2019); doi: $10.1117 / 12.2541326$

Event: Seventh European Workshop on Optical Fibre Sensors, 2019, Limassol, Cyprus 


\title{
Ground-water monitoring using polymer optical fibre Bragg gratings
}

\author{
M. Mehravar ${ }^{\mathrm{a}}$, H. Yang ${ }^{\mathrm{ab}}$, W. Zhang ${ }^{\mathrm{c}}$ and D.J. Webb ${ }^{\mathrm{a} *}$ \\ ${ }^{a}$ School of Engineering and Applied Science, Aston University, Birmingham, UK \\ ${ }^{\mathrm{b}}$ School of Automation Engineering, Northeast Electric Power University, Jilin, China \\ ${ }^{\mathrm{c}}$ Surface Engineering Precision Institute, Cranfield University, Cranfield, UK
}

\begin{abstract}
Optical fibre Bragg grating sensors inscribed in polymer optical fibre have been shown to be sensitive to the water content of the medium surrounding the fibre and this property has been applied, for example, to the monitoring of humidity and the water content of aviation fuel. In this work we assess the feasibility of using such sensors for monitoring the saturation of soils, which is important for civil engineering applications. We find a very non-linear response, with a rapid increase in Bragg wavelength as the water content increases from 0 to $0.5 \%$, with a much more gradual, but still monotonic, increase thereafter. We speculate on the causes of this response.
\end{abstract}

Keywords: optical fibre Bragg grating, FBG, polymer optical fibre, soil, geotechnical monitoring

\section{INTRODUCTION}

Optical fibre Bragg gratings (FBGs) have established themselves as a mature sensing technology over the last 20 years or so and yet they also remain a topic of research, as people seek to exploit non-standard fibre types or develop new applications. An example of this is the development of grating technology in polymer optical fibres ${ }^{1}$, which have different measurand sensitivities compared to silica fibre. Polymer optical fibres (POFs) composed of poly(methyl methacrylate) (PMMA) have an affinity for water, which when absorbed by the fibre causes a swelling accompanied by an increase in refractive index, both of which result in a shift of the Bragg wavelength to higher values. The water absorption is a reversible process with the amount of water in the fibre being determined by the equilibrium relative humidity in the region surrounding the fibre ${ }^{2}$. As well as rendering the polymer optical fibre Bragg grating (POFBG) sensitive to the relative humidity of its environment, this property also makes the POFBG sensitive to the concentration of water containing liquid surrounding the fibre. This is true whether the water is the solvent or the solute; POFBGs have been shown to be highly effective at measuring the very small (10-100 ppm) quantities of water that can dissolve in fuel, for example . $^{3}$

Optical fibre sensors have found application in the geotechnical domain, with a number of researchers exploiting different sensing mechanisms to monitor earth movement to provide a warning of impending landslip; see for example reference [4]. One area that has not so far received much attention is the monitoring of the water content of soil. Soil water content plays a key role in the mechanical properties of soils, affecting the strength and changing the volume of soil that lies above the natural water table. Measuring soil water content is of critical importance to estimate soil effective stress, which is an important parameter in the analysis, design and monitoring of geotechnical engineering structures. Often very small changes in the water content of soil can lead to collapse of the ground and any supported infrastructure resulting in damages to our assets and millions of pounds capital loss. Examples of such events are land sliding, collapse of expansive clays, and sinkholes ${ }^{[6-7] .}$

*d.j.webb@aston.ac.uk

Seventh European Workshop on Optical Fibre Sensors, edited by Kyriacos Kalli, Gilberto Brambilla, Sinead O'Keeffe Proc. of SPIE Vol. 11199, 111991M · C 2019 SPIE · CCC code: 0277-786X/19/\$21 · doi: 10.1117/12.2541326 
In general, due to the complex, tri-phasic (water, air and soil) and non-homogenous nature of soils, the accurate measuring of their water content in the field is one of the greatest challenges that geotechnical engineers face during the site investigation stage of ground works. In this paper we assess the ability of POFBGs as the basis for an effective, accurate and inexpensive approach to measure water content (moisture) in porous media, and in particular in soils. For the first time, the sensitivity of a fibre Bragg grating (FBG) sensor fabricated in polymer optical fibre (POF) to soil water content was investigated. The sensors were tested at various values of gravimetric moisture content up to $20 \%$. The effect of soil temperature was considered and a temperature correction factor was determined. The results show that the sensors, when calibrated correctly, can be a practically useful tool for the measurement of water content with high precision even at low values of water content.

\section{EXPERIMENTAL}

\subsection{Sensor fabrication}

A POFBG was inscribed in a few cm length of single mode, step-index, PMMA based fibre using a HeCd laser and phase mask (see reference [1] for further information). Due to the high attenuation of PMMA at the Bragg wavelength of about $1537 \mathrm{~nm}$, the POF was glued to a silica fibre down-lead and was then fixed under tension to a metallic holder. This was in turn placed inside a metal tube (diameter $=8 \mathrm{~mm}$ ), one end of which was covered by a metallic mesh to prevent sand particles from entering the sensor housing but allow free circulation of air to enable equilibrium to be established between the sensing fibre and surrounding sand; see figure 1. The other end of the tube was sealed. The POFBG was interrogated using a broad band source emitting around $10 \mathrm{~mW}$ across the C-band, with the reflected signal from the POFBG being monitored using an I-Mon spectrometer from Ibsen Photonics.

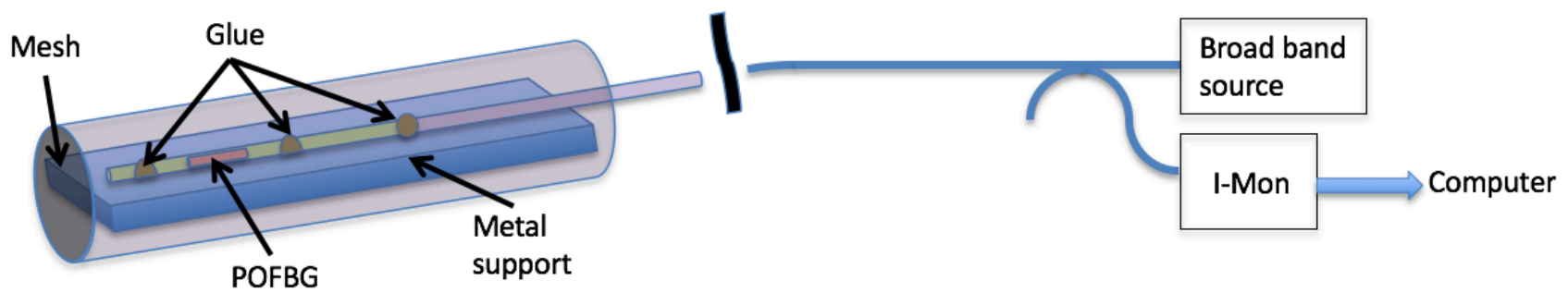

Figure 1. Sensor construction

\subsection{Sample preparation}

All soil samples used in this study were silica sand, which had specific gravity of 2.66 and nominal effective size of $0.63-0.85 \mathrm{~mm}$. The soil samples were oven dried at $105^{\circ} \mathrm{C}$ overnight and then kept in the laboratory environment for at least 24 hours until the soil moisture and temperature equalised with the laboratory condition. In this research, we refer to this state as the 'dry soil' condition - prior to each set of the tests, soil water content was measured in the 'dry soil' condition. In order to generate the predefined gravimetric water content of $0.5,1,5,10,15$ and $20 \%$, water was added to the 'dry soil' and the soil-water mixture was mixed for a duration of 3 minutes using an electrical stand mixer. A cylindrical shape mould with diameter of $12.5 \mathrm{~cm}$ and height of $19 \mathrm{~cm}$ was employed to place the soil in three equal layers. It is worth noting that the compaction of layers (and therefore soil density) was not controlled at this stage and will be the subject of future studies. The POF sensor was inserted into the soil sample to a depth of $5 \mathrm{~cm}$. For the 'dry soil' the sensor was left in the sample for a duration of approximately one hour while for other values of moisture, this was around two and half hours.

\subsection{Sample response}

An example result when moving the sensor between dry and wet (20\%) soil is shown in Figure2. It can be seen that the measured wavelength reaches a constant value before it starts to rise to a new position. The most likely reason for the rise is the gradual movement of water through soil voids due to gravity which results in a variation of moisture content and therefore the measured wavelength. 


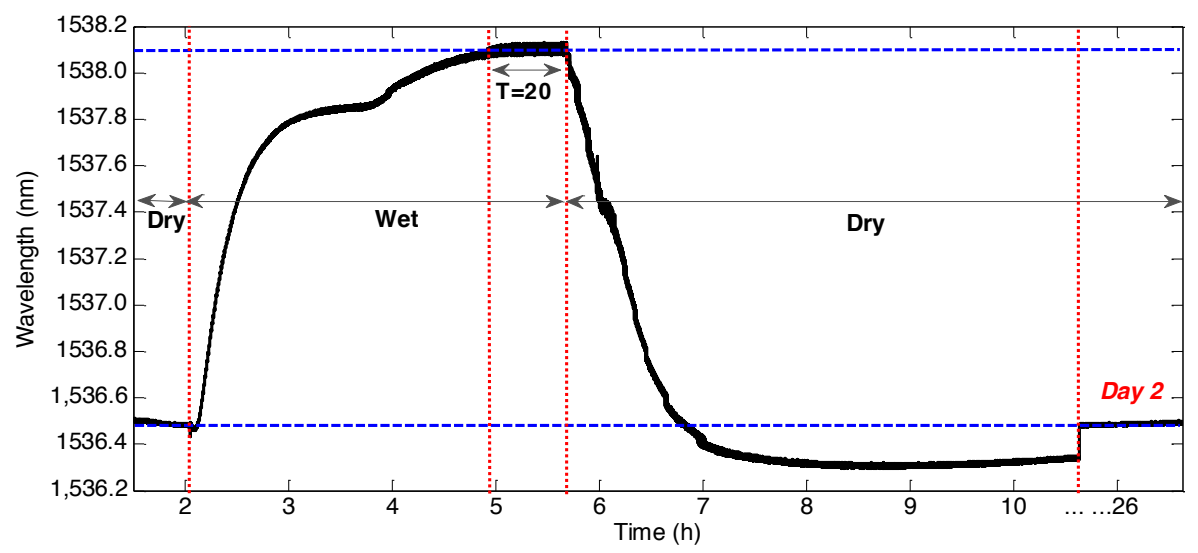

Figure 2. The measured/reflected wavelength from the POF sensor for $20 \%$ soil moisture content at $20.5^{\circ} \mathrm{C}$

\subsection{Temperature}

Because POFBGs have a high sensitivity to temperature, the temperature response of the sensor was determined using an environmental chamber set to a constant humidity of $40 \%$, see Figure 3 . The temperature sensitivity was determined to be $-0.123 \mathrm{~nm} /{ }^{\circ} \mathrm{C}$. This appears much higher than is usually reported for POFBGs due to the fibre being fixed to its metallic support under tension; this prevents any change in fibre length due to thermal expansion, which would otherwise reduce the magnitude of the negative temperature coefficient. The coefficient was used to compensate the sensor reading for laboratory temperature changes.

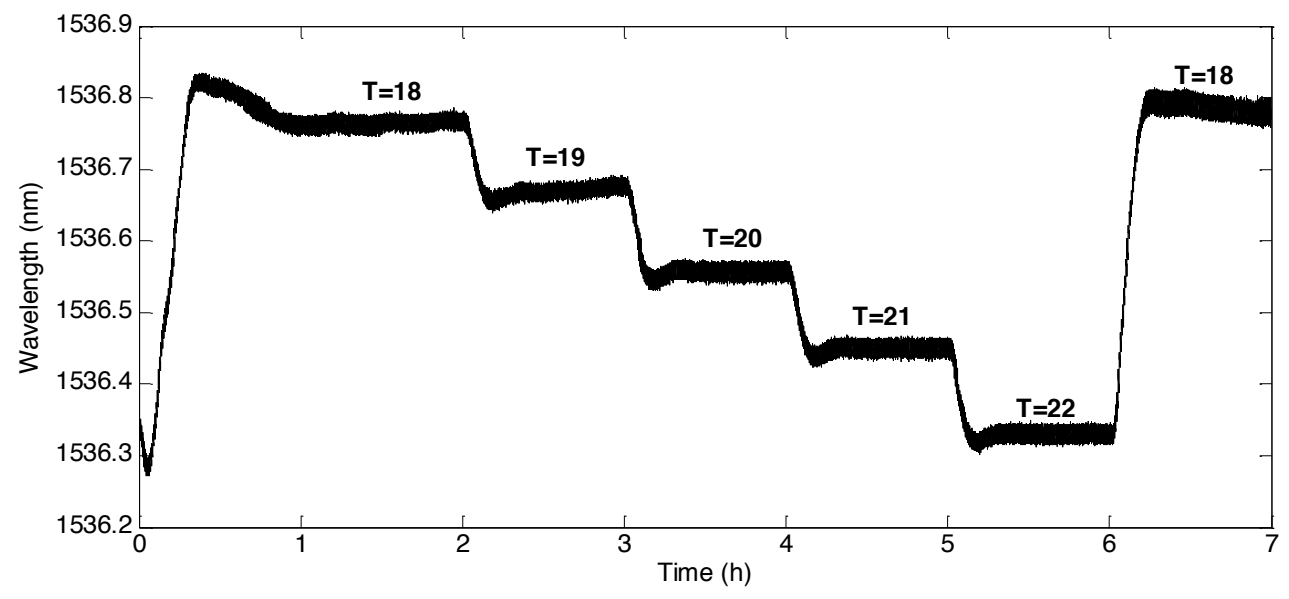

Figure 3. The sensor's sensitivity to a temperature changes in a constant relative humidity of $40 \%$.

\subsection{Summary of results}

Results from different tests ranging from $0.5 \%$ to $20 \%$ water content were used to examine the relationship between recorded wavelength $(\mathrm{nm})$ and soil water content (\%). Figure 4 shows a linear fit together with the data points used to generate the line.

\section{CONCLUSION}

Figure 4 shows an approximately linear relationship and indeed departures from the line may be largely explained by the challenge of knowing the water content at the sensor depth when as stated earlier gravity will gradually change the water content distribution from its initial homogeneous profile. What is not show in Figure 4 however is the large wavelength 
shift from our dry soil to the wet samples shown here. As may be seen from Figure 2, the dry soil produces a sensor reading of $1536.4 \mathrm{~nm}$. The sensor response at very low water contents is still being studied but we believe it relates to the fact that even at low water contents (for example $0.5 \%$ ), the grains of sand are still effectively coated in a layer of water which is likely to lead to a high humidity in the air voids, which is in turn communicated to the POFBG sensor. To conclude, from these initial results, POFBGs look promising for soil water content monitoring, though further work is needed to fully understand the behaviour at very low water contents.

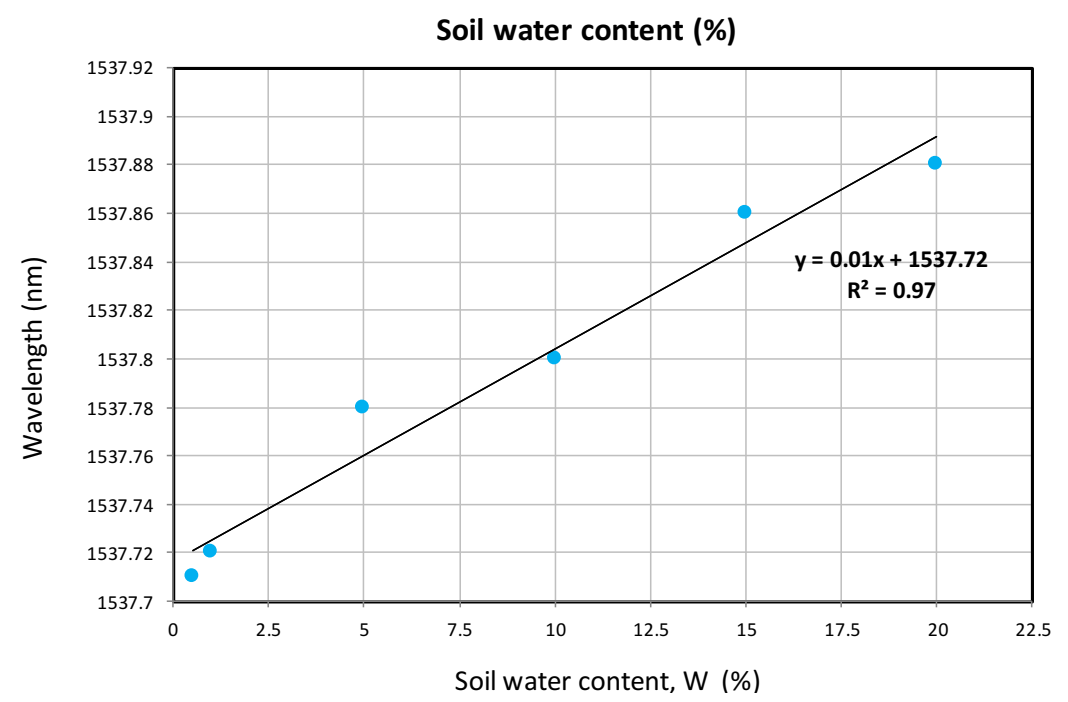

Figure 4. Wavelength $(\mathrm{nm})$ vs. Soil water content (\%)

\section{ACKNOWLEDGEMENT}

Funding for this project by the Aston Institute of Materials Research (AIMR) is greatly acknowledged. The authors would like to thank Prof. Gang-Ding Peng of the University of New South Wales for supply of the polymer fibre.

\section{REFERENCES}

[1] D. J. Webb, "Fibre Bragg grating sensors in polymer optical fibres," Measurement Science and Technology, vol. 26, p. 092004, 2015.

[2] N. G. Harbach, "Fiber Bragg gratings in polymer optical fibers," PhD, EPFL, Lausanne, 2008.

[3] W. Zhang, D. J. Webb, L. Lao, D. Hammond, M. Carpenter, and C. Williams, "Water content detection in aviation fuel by using PMMA based optical fiber grating," Sensors and Actuators B: Chemical, vol. 282, pp. 774-779, 2019.

[4] L. Schenato, L. Palmieri, M. Camporese, S. Bersan, S. Cola, A. Pasuto, et al., "Distributed optical fibre sensing for early detection of shallow landslides triggering," Scientific Reports, vol. 7, p. 14686, 2017/10/31 2017.

[5] Jones, L. D., Jefferson, I., "Expansive soils", Institution of Civil engineers (ICE) Publishing-Manuals series, Chapter C5, 1-46 (2012).

[6] Institution of Civil engineers (ICE), "The state of the nation Infrastructure", (2014). 